Post-print of: Photosynth Res (2011) 110:61-72

\title{
Cytochrome c 6-like protein as a putative donor of electrons to photosystem I in the cyanobacterium Nostoc sp. PCC 7119
}

Francisco M. Reyes-Sosa (1, 2), Jorge Gil-Martínez (1, 3, 2) and Fernando P. Molina-Heredia (1)

(1)

Instituto de Bioquímica Vegetal y Fotosíntesis, Universidad de Sevilla y CSIC, Américo Vespucio 49, 41092 Sevilla, Spain

(2)

Present address: Abengoa Bioenergía Nuevas Tecnologías, Campus Palmas Altas, Energía Solar, no 1, Edificio D, 41012 Sevilla, Spain

(3)

Abengoa Bioenergía Nuevas Tecnologías, Laboratorio ABNT-CITIUS, Reina Mercedes s/n, 41012 Sevilla, Spain

\begin{abstract}
Most organisms performing oxygenic photosynthesis contain either cytochrome c 6 or plastocyanin, or both, to transfer electrons from cytochrome b 6-f to photosystem I. Even though plastocyanin has superseded cytochrome $c$ 6 along evolution, plants contain a modified cytochrome $c 6$, the so called cytochrome $c 6$, whose function still remains unknown. In this article, we describe a second cytochrome c 6 (the so called cytochrome $c 6$-like protein), which is found in some cyanobacteria but is phylogenetically more related to plant cytochrome c $6 \mathrm{~A}$ than to cyanobacterial cytochrome c 6 . In this article, we conclude that the cytochrome c 6-like protein is a putative electron donor to photosystem I, but does play a role different to that of cytochrome c 6 and plastocyanin as it cannot accept electrons from cytochrome $f$. The existence of this third electron donor to PSI could explain why some cyanobacteria are able to grow photoautotrophically in the absence of both cytochrome c 6 and plastocyanin. In any way, the Cyt c 6-like protein from Nostoc sp. PCC 7119 would be potentially utilized for the biohydrogen production, using cell-free photosystem I catalytic nanoparticles.
\end{abstract}

\section{Keywords}

Cytochrome c 6-like protein, Cytochrome c 6A, Biohydrogen, Photosystem I, Spectroscopy, Laser flash spectroscopy

\section{Abbreviations}

Cyt

Cytochrome 
E m

Midpoint redox potential

k bim

Second-order rate constant

$\mathrm{k}$ et

First-order electron transfer rate constant

$k \inf$

Bimolecular rate constant extrapolated to infinite ionic strength

k obs

Observed pseudo-first-order rate constant

PC

Plastocyanin

pl

Isoelectric point

PSI

Photosystem I

\section{Introduction}

Cytochrome (Cyt) c 6 is well known to transfer electrons between Cyt b 6-f complex and photosystem I (PSI) in the thylakoidal lumen of many cyanobacteria and green algae (Hervás et al. 2003a, b). In several organisms, it substitutes for plastocyanin (Pc) under copper deficient conditions. Intensive work has been carried out on Pc and Cyt c 6 to understand their function and how it depends on their structure (e.g., De la Rosa et al. 2002). The surface of the interaction regions of Cyt c 6 and Pc are similar, despite they are structurally unrelated proteins, within any given organism (Ullmann et al. 1997). It has been generally assumed that Pc completely displaced Cyt c 6 in plants along evolution (Kerfeld and Krogmann 1998). However, a modified Cyt c 6, the so called Cyt c 6A, is found in plants and green algae; it is evolutionary related to cyanobacterial Cyt c 6 but is unable to serve as electron donor in the photosynthetic electron transfer chain (Molina-Heredia et al. 2003; Weigel et al. 2003).

The recent sequentiation of genomes of several photosynthetic organisms has allowed the finding of a second Cyt c 6-coding gene in some cyanobacteria, by sequence homology searching with Arabidopsis Cyt c 6A. In the last years, a lot of work has been made to specifically investigate the mechanism of PSI reduction by Pc and Cyt c 6 (reviewed in Díaz-Quintana et al. (2003) and in Fromme et al. (2003)). However, important aspects of the photosynthetic and 
respiratory process in cyanobacteria still remain unknown. In particular, the information concerning the possible involvement of both Pc and Cyt c 6 in the respiratory electron transfer pathway is rather scarce (Navarro et al. 2005), thereby opening the door to the participation of an alternative electron donor to Cyt c oxidase.

It has been described that Synechocystis sp. PCC 6803 is able to grow photoautotrophically in the absence of both Cyt c 6 and Pc (Zhang et al. 1994; Ardelean et al. 2002; Durán et al. 2004; De la Cerda et al. 2007). Several proposals have been made to explain how these cultures can survive in the absence of both Pc and Cyt c 6. Some authors have suggested the existence of a third (but relatively inefficient) electron donor to PSI (Metzger et al. 1995). Some others have proposed the formation of a supercomplex between Cyt b 6-f and PSI to allow direct electron transfer without requirement of any redox carrier (Schmetterer 1994). And others have even discussed the existence of an alternative electron transfer pathway by means of Cyt bd-type (Berry et al. 2002). Under diazotrophic conditions, the assumed arrest of electron flow, at Cyt b 6-f complex level, promotes the uncoupling of both photosystems. To support the high PSI activity during nitrogen fixation, the existence of an alternative electron carrier has been mentioned (Misra et al. 2003). In this article, we describe a second cyanobacterial Cyt c 6 that is evolutionarily related to plant Cyt c 6A. Such a new Cyt c 6 is able to reduce PSI in a relatively efficient way but could not accept electrons from Cyt $\mathrm{f}$, so indicating that it plays another physiological role different from Cyt c 6 and Pc.

\section{Materials and methods}

\section{Bacterial strains and plasmids}

Nostoc sp. PCC 7119 (formerly Anabaena) was described in Adolph and Haerlkorn (1971). Escherichia coli DH5 $\alpha$ (Bethesda Research Laboratories) was used for cloning and plasmid construction. E. coli GM119 (Marinus and Morris 1973) was used for the heterologous expression of both, Cyt c 6 and Cyt c 6 -like protein from Nostoc. Pc was overexpressed in E. coli MC1061 (Casadaban and Cohen 1980). The plasmids pEAC62-lac (this work), pEAC-WT (Molina-Heredia et al. 1998), and pEAP-WT (Molina-Heredia et al. 1998) were used for the overexpression of Cyt c 6-like protein, Cyt c 6, and Pc respectively. The plasmid pEC86 (Arslan et al. 1998), which encodes the E. coli genes required for Cyt c maturation, were used to enhance the expression of Cyt c 6 -like protein and Cyt c 6 in E. coli.

\section{Cloning of the cytA gene from Nostoc sp. PCC 7119}

The direct primer CATCATTATAGGAGCAGCTACATG and the reverse one TTGGGAATTCAAAAGTTTTAACGC, designed from the known sequence of cytA gene (all0161), encoding the Cyt c 6-like protein in Nostoc sp. PCC 7120 (Kaneko et al. 2001), were used to amplify the region containing the cytA gene from the genomic DNA of Nostoc sp. PCC 7119 by the polymerase chain reaction (PCR). The resulting cytA gene was sequenced (The EMBL accession number for the Nostoc sp. PCC 7119 cytA gene is AM902496) and cloned using the TA Cloning Kit Dual Promoter (pCRII) from Invitrogen. To be sure that the sequences did not contained any mutation introduced by PCR, two independent amplifications were carried out and two independent clones of each were sequenced separately. The resulting vector pEAC62-lac was used as expression vector of cytA gene, under the control of the lac promoter. The DNA Sequencing Service from the Spanish National Cancer Research Centre carried out the nucleotide sequence analysis. Other molecular biology protocols used were standard.

\section{Structural modeling}

Structure and surface electrostatic potential of Cyt c 6 and Cyt c 6 -like protein were modeled using the Swiss-Pdb Viewer Program (Guex and Peitsch 1997). The solution structure of Cyt c 6 from the thermophilic cyanobacterium Synechococcus elongatus PCC 6301 (Beissinger et al. 1998) was used as template. Structure of Cyt c 6-like protein from Nodularia spumigena CCY9414 and S. elongatus PCC 6301 were modeled using the program Phyre 
(http://www.sbg.bio.ic.ac.uk/ phyre/) (Kelley and Sternberg 2009). The quality of the modeled structures was tested using the PROCHECK program (Laskowski et al. 1993). The Pc structure used was the solution structure from Anabaena variabilis ATCC 29413 (Badsberg et al. 1996), whose amino acid sequence exhibits 100\% identity with that of Pc from Nostoc sp. PCC 7119 (Molina-Heredia et al. 2001).

\section{Protein expression and purification procedures}

The recombinant Cyt c 6-like protein was expressed in E. coli as described previously for the Nostoc sp. PCC 7119 Cyt c 6 (Molina-Heredia et al. 1998) with some modifications. Purity of the resulting protein fractions was determined using an absorbance ratio A 553/A 275 of ca. 1.0 for pure Cyt c 6-like protein (Molina-Heredia et al. 1998). Protein concentration was determined spectrophotometrically using absorption coefficient of $26.2 \mathrm{mM}-1$ $\mathrm{cm}-1$ at $553 \mathrm{~nm}$ for the reduced protein (this work). Recombinants Cyt c 6 and Pc were expressed and purified as described previously (Molina-Heredia et al. 1998) with the modifications introduced previously (Molina-Heredia et al. 1999, 2001). PSI particles were isolated from Nostoc sp. PCC 7119 cells by $\beta$-dodecyl maltoside solubilization as described previously (Molina-Heredia et al. 1999).

\section{RNA isolation and analysis}

RNA from whole filaments and heterocysts was isolated as described by Muro-Pastor et al. (2002). For Northern analysis, 5 to $10 \mu \mathrm{g}$ of RNA was loaded per lane and electrophoresed in 1\% agarose denaturing formaldehyde gels. Transfer and fixation to Hybond-N+ membranes (Amersham Biosciences) were carried out using $0.1 \mathrm{M} \mathrm{NaOH}$. Hybridization was performed at $65^{\circ} \mathrm{C}$ according to the recommendations of the manufacturers of the membranes. The petJ and cytA probes were fragments of these genes amplified by PCR. All probes were 32P labeled with a Ready-to-Go DNA labeling kit (Amersham Biosciences) using [ $\alpha-32 P] d C T P$. Images of radioactive filters and gels were obtained and quantified with a Cyclone storage phosphor system and OptiQuant image analysis software (Packard).

\section{Analytical methods}

The extinction coefficient and the heme content of the Nostoc sp. PCC 7119 Cyt c 6-like protein at $553 \mathrm{~nm}$ (26.2 mM-1 cm-1) were determined using the pyridine hemochrome method (Appleby 1969). In order to check that the protein was correctly processed, the N-terminus was sequenced in a Procise TM 494 Protein Sequencer (Applied Biosystems). Redox titrations were performed at pH 7.0, in a dual wavelength spectrophotometer as described previously (Molina-Heredia et al. 1998). Isoelectric point (pl) was determined by electrofocusing (Robertson et al. 1987), with a mixture of ampholyte carriers from Bio-Rad, pH range 3-10; the standard proteins used were those of the Sigma isoelectric focusing calibration kit for a $\mathrm{pH}$ range of 6.8-9.3. Molecular weights were determined by MALDI-TOF analysis (Bruker-Daltonics, Germany).

\section{Laser flash-induced kinetics of Nostoc PSI}

Laser flash-induced kinetics of PSI reduction were monitored by following the absorbance changes at $820 \mathrm{~nm}$ as described by Hervás et al. (2003a, b). Unless otherwise stated, the standard reaction mixture contained, in a final volume of $0.2 \mathrm{~mL}, 20 \mathrm{mM}$ buffer (Tricine-KOH, pH 7.5), $0.03 \% \beta$-dodecyl maltoside, an amount of PSI-enriched particles equivalent to $0.36 \mathrm{mg}$ of chlorophyll $\mathrm{ml}-1,0.1 \mathrm{mM}$ methyl viologen, $2 \mathrm{mM}$ sodium ascorbate, $10 \mathrm{mM}$ $\mathrm{MgCl} 2$, and Cyt c 6, Cyt c 6-like protein, or Pc at the indicated concentration. In the studies of the ionic strength effect, the standard reaction mixture contained $30 \mu \mathrm{M}$ of electron donor protein and $\mathrm{NaCl}$ or $\mathrm{MgCl} 2$ at the indicated concentration in Tricine- $\mathrm{KOH}$ buffer, $\mathrm{pH}$ 7.5. All the experiments were performed at $25^{\circ} \mathrm{C}$ in a $1-\mathrm{mm}$ path 
length cuvette. All the kinetic experiments were performed under pseudo-first-order conditions, for which the amount of donor protein was in large excess over the amount of P700+. Each kinetic trace was the average of six measurements with $30 \mathrm{~s}$ spacing between flashes. Oscilloscope traces were treated as a sum of exponential components to calculate the rate constants and amplitudes. Exponential analyses were performed using the Marquardt method with the software devised by Dr. P. Sétif. The error in the observed pseudo-first-order rate constants ( $\mathrm{k}$ obs) was estimated to be less than $10 \%$, based on reproducibility and signal-to-noise ratios. Kinetic analyses were carried out according to the reaction mechanisms proposed by Hervás et al. (1995). Analysis of the dependence of second-order rate constant ( $\mathrm{k} \mathrm{bim}$ ) and extrapolation of estimation of $\mathrm{k}$ bim to infinite ionic strength ( $\mathrm{k}$ inf) was realized according to the formalism developed by Watkins et al. (Watkins et al. 1994).

\section{Results and discussions}

\section{Cloning and expression of the Nostoc Cyt c 6-like protein}

On the basis of the known sequence of cytA gene (all0161), encoding Cyt c 6-like protein in Nostoc sp. PCC 7120 (Kaneko et al. 2001), a couple of oligonucleotides outside the ORF (see materials and methods) were designed. This allowed us the cloning of the complete gene by PCR amplification. Just a DNA band was amplified, recovered, and ligated using the pCRII cloning kit. The plasmid with the cytA gene in phase with lac promoter was used as protein expression vector (pEAC62-lac). The sequence of the cytA gene from Nostoc sp. PCC 7119 (EMBL accession number AM902496) turned out to be identical to the one of the strain 7120 , as also identical are other genes involved in the photosynthetic electron transfer chain, namely petJ, petE (Molina-Heredia et al. 1998), and petA (Albarrán et al. 2005).

The Nostoc Cyt c 6-like protein was expressed in E. coli and purified using the same experimental protocol that we have described previously for the Nostoc sp. PCC 7119 Cyt c 6 (Molina-Heredia et al. 1998), with some modifications. E. coli GM119 was used as expression strain, and the co-expression with the plasmid pEC86 (see materials and methods) was made to enhance the Cyt production. $20 \mathrm{mg}$ of the Cyt c 6 -like protein was extracted from the periplasmic fraction of 10-L E. coli cell cultures, and $8 \mathrm{mg}$ of pure Cyt c 6-like protein, with an A 553/A 275 ratio for the reduced/oxidized protein of 1.5, was finally obtained (see Fig. 1). MALDI-TOF analysis and sequentiation of the $\mathrm{N}$-terminus of the purified protein suggest that $\mathrm{E}$. coli rightly processed the heterologous protein and the transit peptide was properly removed at the processing site ANA-ENT (Fig. 2a). Table 1 shows the physicochemical parameters of the mature protein.

\section{UV/Visible absorption spectra}

The UV/Visible absorption spectrum of the purified Nostoc Cyt c 6-like protein showed typical spectroscopic features of $c$ type Cyts and was similar to that of Nostoc Cyt c 6 (Fig. 1). In the oxidized state, the purified Nostoc Cyt c 6-like protein exhibits a broader band with a maximum at $528 \mathrm{~nm}$, a characteristic absorbance maximum at $409 \mathrm{~nm}$ ( $\gamma$, or Soret), and two bands at 359 and $280 \mathrm{~nm}$ (protein), respectively. Upon reduction with an excess of sodium dithionite, the Soret band shifts to $417 \mathrm{~nm}$, and appears the characteristic absorbance maxima at $553(\alpha)$ and $523 \mathrm{~nm}(\beta)$. In addition, in both, Cyt c 6-like and Cyt c 6 from Nostoc sp. PCC 7119, $\alpha$ band maximum is $553 \mathrm{~nm}$ and extinction coefficient at this wavelength is the same.

\section{Sequence analysis}


As shown in Fig. 2a, the transit peptide from the Nostoc Cyt c 6-like protein is similar to the one of Cyt c 6 . The cellular localization of the Nostoc Cyt c 6 -like protein is a key point to bear in mind when assessing its physiological role. For this, the close similarity between the transit peptides of both Cyts and the sequence analysis performed with SignalP 3.0 Server (Emanuelsson et al. 2007), DAS (Cserzo et al. 1997), and PSORTb (Yu et al. 2010), suggests that the Cyt c 6 -like protein could, in fact, be located inside the thylakoidal lumen, as are Cyt c 6 and Pc. In addition, as we have already discussed, the Cyt c 6 -like protein was properly processed by $\mathrm{E}$. coli, and the transit peptide was correctly removed at the processing site ANA-ENT, and the mature protein was located at the periplasmic space of E. coli that is the cellular compartment equivalent to the thylakoidal lumen from cyanobacteria.

As we have already described, the Cyt c 6 from Nostoc sp. PCC 7119 interacts with both Cyt $\mathrm{f}$ and PSI using the same surface regions (Molina-Heredia et al. 1999; Díaz-Moreno et al. 2005). These regions are site 1 (or the so called north hydrophobic pole) and site 2 (or the so called east face). Site 1 is a hydrophobic region, around the solvent-exposed heme propionates, that resembles the north pole of Pc (Frazão et al. 1995), providing the contact surface for electron transfer. Site 2, which is a charged patch, is similar to the east face of eukaryotic Pc (Frazão et al. 1995), driving the electrostatic movement toward its two membrane-anchored partners. In addition, we have previously used NMR transverse relaxation-optimized spectroscopy in solution to analyze the physiological interaction between PSI, both as a monomer (340 kDa) and a trimer (1020 kDa), with its physiological reaction partner Cyt c 6 from Nostoc sp. PCC 7119. Upon titration of the photosystem into Cyt c 6, the residues on the surface of Cyt c 6 that are affected by the addition of PSI are: 19, S11, N13, L24, V25, and K29, at the hydrophobic interaction site, and A57, M58, A60, F61, K62, R64, L65, and K66 at the basic electrostatic site (Díaz-Moreno et al. 2005). As we can see in Fig. 2b, both regions are highly conserved in Cyt c 6-like proteins. The hydrophobic site presents a $50 \%$ of identity and a $100 \%$ of homology between the two proteins, and the electrostatic site presents a $62 \%$ of both, identity and homology. The most remarkable fact is that in the Cyt c 6 -like protein the electrostatic region is less charged than in Cyt c 6, and that in the Cyt c 6-like protein appears two arginyl residues near the hydrophobic site. With these data, we could think than the Cyt $\mathrm{c} 6$-like protein would be capable of interacting with the same partners that Cyt c 6 , but such interaction would have to be weaker.

Figure $2 \mathrm{~b}$ shows an alignment of three Cyt $\mathrm{c} 6$-like proteins with some other proteins belonging to the two more related groups, the Cyt c 6 and the Cyt c 6A. The Cyt c 6 -like protein sequences have a high identity and homology of ca. 69 and 78\%, respectively, thus indicating that the Cyt c 6-like protein is a highly conserved protein that displays a variability, among different organisms, much smaller that the Cyt c 6 group. Like Cyt c 6 and Cyt c 6A, Cyt c 6-like protein has the arginyl residue 64; that is strictly conserved in all Cyt c 6 sequences (Molina-Heredia et al. 2001). We have previously described that Pc has an equivalent arginyl residue and this single residue in both, Cyt $c$ 6 and Pc, is required for efficient reduction of PSI (Molina-Heredia et al. 2001). In Cyt c 6 and Cyt c 6A only a single arginyl residue appears, however, it is interesting to remark that in the Cyt c 6-like protein it shows two more arginines in position 26 and 27 that are also conserved.

In order to elucidate its evolutionary origin, we have compared the amino acid sequences of Cyt c 6-like protein with those of the five other soluble monoheme Cyts with His-Met axial coordination of photosynthetic organisms: Cyt c 6 (cyanobacteria and chloroplasts of green algae), Cyt c 6A (chloroplasts of plants and green algae), Cyt c M (cyanobacteria), Cyt c 2 (non-oxygenic bacteria), and respiratory Cyt c (mitochondria of algae and plants). As can be seen in Fig. 3, all Cyt c 6 -like proteins constitute by themselves a different clade, as they are all in a single branch that is independent of the other heme proteins. Also, Bialek et al. (2008) described two well-defined groups of cyanobacterial Cyt c 6-like proteins, the Cyt c 6B and the Cyt c 6C. The Cyt c 6 -like protein described here falls into the Cyt c 6 C group. Cyt c 6 seems indeed to sequentially evolve in a linear branch from prokaryotes to eukaryotic 
algae, whereas both Cyt c 6-like protein groups and Cyt c 6A would follow another pathway that had previously diverged from Cyt c 6.

\section{Gene expression analysis}

The intracellular levels of Cyt c 6 and Pc are regulated by the amount of copper in the culture medium (Bovy et al. 1992). Cyt c 6 is dominant under copper-depleted conditions, whereas Pc becomes dominant when the medium contains an adequate concentration of copper. We have studied the expression of the cytA gene in comparison with the petJ gene that encodes Cyt c 6 . This study has been performed in cyanobacterial cells grown under standard culture conditions, with or without copper and with or without nitrate in the growth medium. In this case, we detected the cytA transcript under all conditions, also in heterocysts, and it does not seem to have a specific regulation by copper. In fact, our results showed that the expression of cytA gene was about 3-fold lower than petJ gene, in copper-depleted conditions. In diazotrophic conditions, the expression of cytA gene was 1.8 and 2.1-times lower than petJ gene, in vegetative and heterocyst cells, respectively.

\section{Midpoint redox potential}

In the photosynthetic electron flow from Nostoc sp. PCC 7119, Cyt c 6 and Pc, whose E m at pH 7.0 are +337 and +355 mV respectively (Molina-Heredia et al. 1998), take electrons from Cyt $\mathrm{f}$, whose $\mathrm{E} \mathrm{m}$, at the same $\mathrm{pH}$ 7.0, is +334 mV (Albarrán et al. 2005), and donate them to the photo-oxidized PSI, whose E m (P700) is $+500 \mathrm{mV}$ (Brettell 1997). The $\mathrm{E} \mathrm{m}$ at pH 7.0 of the Nostoc Cyt c 6-like protein is $+199 \mathrm{mV}$ (Table 1), which is ca. $135 \mathrm{mV}$ lower than that of Cyt c 6, Pc, and Cyt $\mathrm{f}$. This $\mathrm{E} \mathrm{m}$ is different, but closer to the redox potentials reported for other related Cyts at the same $\mathrm{pH}$, as Cyt c M, whose $\mathrm{E} \mathrm{m}$ is $+150 \mathrm{mV}$ (Molina-Heredia et al. 2002), or Cyt c 6A, with an E m of $+140 \mathrm{mV}$ (Molina-Heredia et al. 2003). With this $\mathrm{E} \mathrm{m}$ it is clear that the Cyt c 6 -like protein cannot replace Cyt c 6 and Pc, in the electron transfer between Cyt $f$ and PSI, because from a thermodynamic point of view it is difficult to see how the Cyt c 6-like protein would accept electrons from Cyt $f$ against potential. Similar results have been described for the PetJ2 protein, found in the cyanobacterium Synechococcus sp. PCC 7002 (Bialek et al. 2008), that is one other Cyt c 6-like protein. However, this fact does not discard the possibility that the Nostoc Cyt c 6-like protein could donate electrons to the PSI or terminal oxidases, opening the likelihood of a different route for photosynthetic or respiratory electron transfer. Recently, it has been described in the non-heterocystous nitrogen fixing cyanobacterium Plectonema boryanum that, during nitrogen fixation, the arrest of photosynthetic electron flow at Cyt b 6-f site leads to the uncoupling of the two photosystems and that the high PSI activity during light-dependent nitrogen fixation is supported by an alternative electron donor system (Misra et al. 2003). Heterocysts from Nostoc lack PSII, and only PSI activity supports the light-dependent nitrogen fixation. Thus, a plausible function of the Cyt c 6-like protein could be the reduction of the PSI in the heterocysts during light-dependent nitrogen fixation, transferring electrons from any oxidative catabolic process to PSI.

\section{pl and electrostatic surface potential distribution}

The pls of Cyt c 6 and Pc show wide variations among different photosynthetic organisms, but are very similar to each other within any strain (De la Rosa et al. 2002). Thus, in Nostoc sp. PCC 7119, both proteins are basic with a pl of 9.0 and 8.8, respectively (Molina-Heredia et al. 1998). The pl value of the Cyt c 6-like protein experimentally determined in this study is 8.0, which is close to that described for Cyt c 6 and Pc.

Also, we have estimated the pl of other two Cyt c 6-like proteins from N. spumigena CCY9414 (EMBL accession number A0ZCV9) and S. elongatus PCC 6301 (EMBL accession number Q5N1R2) using the program ProtParam (Gasteiger et al. 2005). The estimated pl value of both proteins is 8.7, close to that of Nostoc sp. PCC 7119. As the 
Nostoc proteins, N. spumigena CCY9414 Cyt c 6 and Pc are basic, with a pl value of 9.2 and 8.7, respectively; however, in S. elongatus PCC 6301 both proteins are acidic, with a pl value of 5.6 and 4.7. Whereas pl values of Cyt c 6 and Pc are very similar to each other within the same organism (De la Rosa et al. 2002), the pl value of Cyt c 6like protein seems to be similar among different photosynthetic organisms and no necessarily correlated whit Cyt $c$ 6 and Pc. These findings might be due to the fact that Cyt c 6 -like protein could have a different function, responsible for a conserved $\mathrm{pl}$ value in all studied cyanobacteria.

The $\mathrm{pl}$ of a metalloprotein measures the global charge of the molecule, however, not only the global charge, which defines the long-range movements, but also the surface potential distribution, which controls the short-range interactions, is critical for right orientation and complex formation. Thus, the surface electrostatic potential distribution of Cyt c 6, Pc, and Cyt c 6-like protein from Nostoc sp. PCC 7119 and Cyt c 6-like protein from N. spumigena CCY9414 and S. elongatus PCC 6301 were calculated. As shown in Fig. 4, Cyt c 6 and Pc from Nostoc present a positively charged site 2 that, in fact, plays the same functional role (Molina-Heredia et al. 2001). If the Cyt c 6-like protein could replace both proteins, it must have a site 2 equivalent to that of the two other metalloproteins; however, site 2 from the Cyt c 6 -like protein is negatively charged. Much more interesting is the comparison between the surface electrostatic potential distributions from the Nostoc Cyt c 6-like protein with those from others related Cyt c 6-like proteins. As it is shown in Fig. 4, the three Cyt c 6-like proteins have a similar surface distribution of charges and, in all cases, a clearly negatively charged site 2 appears. As we have mentioned before, Cyt c 6 and Pc have undergone a process of convergent evolution to make the same function resulting similar surfaces in both proteins within the same organism, but a different one from one organism to another (De la Rosa et al. 2002). Nevertheless, the tree Cyt c 6 -like proteins compared here seems to have undergone another type of evolution, by a different way from Cyt c 6 and Pc, which has resulted in a putative conserved protein electrostatic distribution surface. However, as we have already discussed in the "Sequence analysis", site 1, responsible for the interactions at short distance and for the correct positioning of the redox centers, seems to be more conserved, so it cannot be discarded as a reacting partner of PSI.

\section{Laser flash-induced kinetics of Nostoc PSI}

The relatively low $\mathrm{E} m$ of the Nostoc Cyt c 6 -like protein renders it as a very inefficient electron acceptor from Cyt $f$ $(\mathrm{E} \mathrm{m}=+334 \mathrm{mV})$, but it might be expected to stimulate the reaction with PSI, in accordance with Marcus theory (Marcus and Sutin 1985). In fact, the Nostoc Cyt c 6-like protein was found to react with PSI 3.8 times slower than Cyt c 6, and only 2.4 times slower than Pc (see Table 1), under the experimental conditions described in Fig. 5. The kinetics of PSI reduction by Cyt c 6 are biphasic, but those with the Cyt c 6 -like protein and Pc are monoexponential, lacking the fast phase typically observed with Cyt c 6 . This can be interpreted by assuming that there is no formation of any stable complex between PSI and the Cyt c 6-like protein and thus the reaction follows a collisional kinetic mechanism (type I) (Hervás et al. 1995).

Taking into account the electrostatic nature of the interaction of Cyt c 6 and Pc with PSI, a detailed analysis of the effect of ionic strength on the second-order rate constant for PSI reduction ( $k$ bim) was performed. Figure 6 shows that the $\mathrm{k}$ bim values with Cyt c 6 and Pc monotonically diminish with increasing $\mathrm{NaCl}$ concentration, thereby indicating the existence of attractive electrostatic interactions between the reaction partners as previously described by Hervás et al. (1995). However, in the Cyt c 6-like protein the attractive electrostatic interaction with PSI rather insignificant as inferred from the fact that there is practically no dependence of $\mathrm{k}$ bim on ionic strength. These results are in support that the Cyt c 6 -like protein cannot substitutes for Cyt c 6 and Pc in the electronic flow between Cyt b 6-f and PSI. The k bim values with the Cyt c 6-like protein are lower than those with Cyt c 6 and Pc, mainly under conditions of very low ionic strength, (in these no physiological conditions the electrostatic 
interactions are magnified), indicating that the important changes observed in the surface electrostatic potential distribution at site 2 of the Cyt c 6 -like protein (see Fig. 4) alter drastically the attractive electrostatic interactions between PSI and the Cyt c 6-like protein. As it has been discussed before in the "Sequence analysis", this is in agreement with the fact that the residues in site 2 required for the electrostatic interactions are not conserved. Using the Watkins equation (Watkins et al. 1994), the bimolecular rate constant extrapolated to infinite ionic strength ( $\mathrm{k}$ inf) (which facilitates the analysis of the intrinsic reactivity of redox partners in the absence of electrostatic interactions) can be calculated from the experimental data. As shown in Table 1, the $k$ inf values with the Cyt c 6-like protein are very similar to that with $\mathrm{Pc}$, and of the same magnitude order than the one with Cyt c 6 , a fact that can be explained by assuming that, as it has been discussed before in the "Sequence analysis", the site 1, responsible of the hydrophobic interaction region of the Cyt c 6-like protein, providing the contact surface for electron transfer with PSI, presents a $50 \%$ of identity and a $100 \%$ of homology with those from Cyt c 6 . From a physiological point of view is much more interesting to compare the $\mathrm{k}$ bim under conditions of high ionic strength and the $\mathrm{k}$ inf, because in vivo the ionic strength inside the thylakoid is $0.2-0.3 \mathrm{M}$, the presence of the thylakoid membrane imposes additional electrostatic and hydrophobic interactions, along with a physical diffusional constraining factor, and the molecular crowding inside the cell can also impose severe limitations on the diffusion and encounter of protein partners. Due to this, others authors have been found relevant differences and discrepancies between the in vitro and in vivo PSI reduction kinetics, mainly regarding the role played by the electrostatic interactions (Durán et al. 2006; Finazzi et al. 2005; Soriano et al. 1998; Sommer et al. 2004).

Divalent cations like Mg2+ have previously been reported to be specifically involved in Pc/PSI interactions in other organisms, but not in Nostoc sp. PCC 7119 (Hervás et al. 1995). Thus, an analysis of the effect of Mg2+ cations on the bimolecular rate constant of PSI reduction by Cyt c 6-like protein was performed. The experimental results show that the bimolecular rate constant exhibits a biphasic dependence on ionic strength (Fig. 6). To low ionic strength, the bimolecular rate constant increases after the addition of small amounts of $\mathrm{Mg} 2+$, and later remains constant, but significantly greater than without Mg. This indicates that it can have small electrostatic repulsions between PSI and the Cyt c 6-like protein, repulsions that are screened by the presence of divalent cations. According to the formalism by Watkins et al. (1994), such biphasic dependence could be explained by assuming that the monopolar and dipolar energy terms are of opposite sign. In addition, we can see that the Cyt c 6-like protein reacts with PSI less efficiently than Cyt c 6 and Pc, and that such collisional reaction is mainly directed by short distant hydrophobic interactions.

\section{Conclusions}

We have cloned and overexpressed the cytA (all0161) gene from Nostoc sp. PCC 7119, which encodes for a Cyt c 6like protein. The sequence analysis showed that the Cyt c 6 -like protein is located inside the thylakoidal lumen, and that possesses a 100\% homology with the Cyt c 6 hydrophobic interaction region with PSI and Cyt $f$ (site 1). At the basis of the phylogenetic analysis, the Cyt c 6 -like protein from Nostoc falls into the Cyt c 6 C group that is different to those from Cyt c 6. Cyt c 6 seems indeed to sequentially evolve in a linear branch from prokaryotes to eukaryotic algae, whereas cyanobacterial Cyt c 6-like protein groups (B and C) and plant Cyt c 6A would follow another pathway that had previously diverged from Cyt c 6 . The pl values, surface electrostatic potential distribution, and the E $\mathrm{m}$ suggest that the Cyt c 6-like protein would not accept electrons from Cyt f. However, the Nostoc Cyt c 6-like protein was found to react with PSI 3.8 times slower than Cyt c 6, and only 2.4 times more slowly than Pc. In addition, physico-chemical and functional analysis showed that the Cyt c 6 -like protein is a relatively low efficient electron donor to PSI, but they cannot replace Cyt $c 6$ and Pc as electron transfer between Cyt $f$ and PSI. 
From an applied point of view, the Nostoc Cyt c 6-like protein would be potentially utilized for the hydrogen production, using cell-free PSI catalytic nanoparticles. Recently, Iwuchukwu et al. (2010) have showed that selforganized plasticization of PSI nanoparticles allows electron transport from sodium ascorbate to PSI via Cyt c 6 and finally to the platinum catalyst, where hydrogen is formed. The Noctoc Cyt c 6-like protein reacts with PSI and is relatively efficient to high ionic strength in the presence of divalent cations (Fig. 6). For that reason, the Nostoc Cyt c 6-like protein could be used, alone or in combination with Cyt c 6 or Pc, as soluble electron carrier in such selforganized photosynthetic nanoparticles for cell-free hydrogen production.

In addition, the existence of a potential third electron donor to PSI could explain why a cyanobacterium as Synechocystis sp. PCC 6803 is able to grow photoautotrophically in the absence of Cyt c 6 and Pc, and is in agreement with the previous results that suggest the existence of a third (bud relatively inefficient) electron donor to PSI (Metzger et al. 1995). Therefore, we propose that the Nostoc Cyt c 6-like (Cyt c 6C) protein is an alternative photosynthetic soluble electron donor to PSI, which probably acts in an electron transfer pathway different to those from Cyt c 6 and Pc.

\section{Acknowledgments}

This research was supported by the Dirección General de Investigación Científica y Técnica (DGICYT, Grant BFU2006-01361/BMC) and Junta de Andalucía (PAI, BIO022 and BIO198). We are very grateful to Prof. M. Hervás and Dr. J.A. Navarro for critically reading the manuscript. We also are grateful to Pilar Alcántara for its technical assistance. 


\section{References}

Adachi J, Hasegawa M (1992) MOLPHY version 2.3: Programs for Molecular Phylogenetics Based on Maximum Likelihood. In: Computer Science Monographs 28. Institute of Statistical Mathematics, Tokyo

Adolph KW, Haerlkorn R (1971) Isolation and characterization of a virus infecting the blue-green alga Nostoc muscorum. Virology 46:200-208. doi:10.1016/0042-6822(71)90023-7

Albarrán C, Navarro JA, Molina-Heredia FP, PdelS Murdoch, De la Rosa MA, Hervás M (2005) Laser flash-induced kinetic analysis of cytochrome $\mathrm{f}$ oxidation by wild-type and mutant plastocyanin from the cyanobacterium Nostoc sp. PCC 7119. Biochemistry 44:11601-11607. doi:10.1021/bi050917g

Appleby CA (1969) Electron transport systems of Rhizobium japonicum. II. Rhizobium haemoglobin, cytochromes and oxidases in free-living (cultured) cells. Biochim Biophys Acta 172:88-105. doi:10.1016/0005-2728(69)90094-2

Ardelean I, Matthijs HC, Havaux M, Joset F, Jeanjean R (2002) Unexpected changes in photosystem I function in a cytochrome c 6-deficient mutant of the cyanobacterium Synechocystis PCC 6803. FEMS Microbiol Lett 213:113119. doi:10.1111/j.1574-6968.2002.tb11294.x

Arslan E, Schulz H, Zufferey R, Künzler P, Thöny-Meyer L (1998) Overproduction of the Bradyrhizobium japonicum ctype cytochrome subunits of the cbb 3 oxidase in Escherichia coli. Biochem Biophys Res Commun 251:744-747. doi:10.1006/bbrc.1998.9549

Badsberg U, Jorgensen AMM, Gesmar H, Led JJ, Hammerstad JM, Jespersen LL, Ulstrup J (1996) Solution structure of reduced plastocyanin from the blue-green alga Anabaena variabilis. Biochemistry 35:7021-7031. doi:10.1021/bi960621y

Beissinger M, Sticht H, Sutter M, Ejchart A, Haehnel W, Rosch P (1998) Solution structure of cytochrome c 6 from the thermophilic cyanobacterium Synechococcus elongates. EMBO J 17:27-36. doi:10.1093/emboj/17.1.27

Berry S, Schneider D, Vermaas WFJ, Rogner M (2002) Electron transport routes in whole cells of Synechocystis $\mathrm{sp}$. PCC 6803: the role of the cytochrome bd-type oxidase. Biochemistry 4:3422-3429. doi:10.1007/s11120-008-9398-7

Bialek W, Nelson M, Tamiola K, Kallas T, Szczepaniak A (2008) Deeply branching c 6-like cytochromes of cyanobacteria. Biochemistry 47:5515-5522. doi:10.1021/bi701973g

Bovy A, de Vrieze G, Borrias M, Weisbeek P (1992) Transcriptional regulation of the plastocyanin and cytochrome c553 genes from the cyanobacterium Anabaena species PCC 7937. Mol Microbiol 6:1507-1513. doi:10.1111/j.13652958.1992.tb00871.x

Brettell K (1997) Electron transfer and arrangement of the redox cofactors in photosystem I. Biochim Biophys Acta 1318:322-373. doi:10.1016/S0005-2728(96)00112-0

Casadaban MJ, Cohen SN (1980) Analysis of gene control signals by DNA fusion and cloning in Escherichia coli. J Mol Biol 138:179-207. doi:10.1016/0022-2836(80)90283-1

Cserzo M, Wallin E, Simon I, von Heijne G, Elofsson A (1997) Prediction of transmembrane alpha-helices in prokaryotic membrane proteins: the Dense Alignment Surface method. Protein Eng 10:673-676. doi:10.1093/protein/10.6.673 
De la Cerda B, Castielli O, Durán RV, Navarro JA, Hervás M, De la Rosa MA (2007) A proteomic approach to iron and copper homeostasis in cyanobacteria. Brief Funct Genomic Proteomic 6:322-329. doi:10.1093/bfgp/elm030

De la Rosa MA, Navarro JA, Díaz-Quintana A, De la Cerda B, Molina-Heredia FP, Balme A, Murdoch PS, Díaz-Moreno I, Durán RV, Hervás M (2002) An evolutionary analysis of the electrostatic interactions of photosystem I with cytochrome c 6 and plastocyanin. Bioelectrochemistry 55:41-45. doi:10.1016/S1567-5394(01)00136-0

Díaz-Moreno I, Díaz-Quintana A, Molina-Heredia FP, Nieto PM, Hansson O, De la Rosa MA, Karlsson BG (2005) NMR analysis of the transient complex between membrane photosystem I and soluble cytochrome c 6 . J Biol Chem 280:7925-7931. doi:10.1074/jbc.M412422200

Díaz-Quintana A, Navarro JA, Hervás M, Molina-Heredia FP, De la Cerda B, De la Rosa MA (2003) A comparative structural and functional analysis of cyanobacterial plastocyanin and cytochrome $c 6$ as alternative electron donors to photosystem I. Photosynth Res 75:97-110. doi:10.1023/A:1022841513592

Durán RV, Hervás M, De La Rosa MA, Navarro JA (2004) The efficient functioning of photosynthesis and respiration in Synechocystis sp. PCC 6803 strictly requires the presence of either cytochrome c 6 or plastocyanin. J Biol Chem 279:7229-7233. doi:10.1074/jbc.M311565200

Durán RV, Hervás M, De la Cerda B, De la Rosa MA, Navarro JA (2006) A laser flash-induced kinetic analysis of in vivo photosystem I reduction by site-directed mutants of plastocyanin and cytochrome c 6 in Synechocystis sp. PCC 6803. Biochemistry 45:1054-1060. doi:10.1021/bi052090w

Emanuelsson O, Brunak S, von Heijne G, Nielsen H (2007) Locating proteins in the cell using TargetP, SignalP and related tools. Nat Protoc 2:953-971. doi:10.1038/nprot.2007.131

Finazzi G, Sommer F, Hippler M (2005) Release of oxidized plastocyanin from photosystem I limits electron transfer between photosystem I and cytochrome b $6 \mathrm{f}$ complex in vivo. Proc Natl Acad Sci USA 102:7031-7036. doi:10.1073/pnas.0406288102

Frazão C, Soares CM, Carrondo MA, Pohl E, Dauter Z, Wilson KS, Hervás M, Navarro JA, De la Rosa MA, Sheldrick GM (1995) Ab initio determination of the crystal structure of cytochrome $c 6$ and comparison with plastocyanin. Structure 3:1159-1169. doi:10.1016/S0969-2126(01)00252-0

Fromme P, Melkozernov A, Jordan P, Krauss N (2003) Structure and function of photosystem I: interaction with its soluble electron carriers and external antenna systems. FEBS Lett 555:40-44. doi:10.1016/S0014-5793(03)01124-4

Gasteiger E, Hoogland C, Gattiker A, Duvaud S, Wilkins MR, Appel RD, Bairoch A (2005) Protein identification and analysis tools on the ExPASy server. In: Walker JM (ed) The proteomics protocols handbook. Humana Press Inc, Totowa, NJ, pp 571-607

Guex N, Peitsch MC (1997) SWISS-MODEL and the Swiss-PdbViewer: an environment for comparative protein modelling. Electrophoresis 18:2714-2723. doi:10.1002/elps.1150181505

Hervás M, Navarro JA, Díaz A, Bottin H, De la Rosa MA (1995) Laser-flash kinetic analysis of the fast electron transfer from plastocyanin and cytochrome c 6 to photosystem I. Experimental evidences on the evolution of the reaction mechanism. Biochemistry 34:11321-11326. doi:10.1021/bi00036a004 
Hervás M, Myshkin E, Vintonenko N, De la Rosa MA, Bullerjahn GS, Navarro JA (2003a) Mutagenesis of Prochlorothrix plastocyanin reveals additional features in Photosystem I interaction. J Biol Chem 278:8179-8183. doi:10.1074/jbc.M211913200

Hervás M, Navarro JA, De la Rosa MA (2003b) Electron transfer between membrane complexes and soluble proteins in photosynthesis. Acc Chem Res 36:798-805. doi:10.1021/ar020084b

Iwuchukwu IJ, Vaughn M, Myers N, O'Neil H, Frymier P, Bruce BD (2010) Self-organized photosynthetic nanoparticle for cell-free hydrogen production. Nat Nanotechnol 5:73-79. doi:10.1038/nnano.2009.315

Kaneko T, Nakamura Y, Wolk CP, Kuritz T, Sasamoto S, Watanabe A, Iriguchi M, Ishikawa A, Kawashima K, Kimura T, Kishida $Y$, Kohara M, Matsumoto M, Matsuno A, Muraki A, Nakazaki N, Shimpo S, Sugimoto M, Takazawa M, Yamada M, Yasuda M, Tabata S (2001) Complete genomic sequence of the filamentous nitrogen-fixing cyanobacterium Anabaena sp. strain PCC 7120. DNA Res 8:205-213. doi:10.1093/dnares/8.5.205

Kelley LA, Sternberg MJE (2009) Protein structure prediction on the web: a case study using the Phyre server. Nat Protoc 4:363-371. doi:10.1038/nprot.2009.2

Kerfeld CA, Krogmann DW (1998) Photosynthetic cytochromes c in cyanobacteria, algae and plants. Annu Rev Plant Physiol Plant Mol Biol 49:397-425. doi:10.1146/annurev.arplant.49.1.397

Laskowski RA, McArthur MW, Moss DS, Thornton JM (1993) PROCHECK: a program to check the stereochemical quality of protein structures. J Appl Crystallogr 26:283-291. doi:10.1107/S0021889892009944

Marcus RA, Sutin N (1985) Electron transfers in chemistry and biology. Biochim Biophys Acta 811:265-322. doi:10.1016/0304-4173(85)90014-X

Marinus MG, Morris NR (1973) Isolation of deoxyribonucleic acid methylase mutants of Escherichia coli K-12. J Bacteriol 114:1143-1150

Metzger SU, Pakrasi HB, Whitmarsh J (1995) Characterization of a double deletion mutant that lacks cytochrome c 6 and cytochrome c M in Synechocystis 6803. In: Mathis P (ed) Photosynthesis: from light to biosphere. Kluwer Academic Publishers, Dordrecht, pp 823-826

Misra HS, Khairnar NP, Mahajan SK (2003) An alternate photosynthetic electron donor system for PSI supports light dependent nitrogen fixation in a non-heterocystous cyanobacterium, Plectonema boryanum. J Plant Physiol 160:33-39. doi:10.1078/0176-1617-00846

Molina-Heredia FP, Hervás M, Navarro JA, De la Rosa MA (1998) Cloning and correct expression in Escherichia coli of the petE and petJ genes respectively encoding plastocyanin and cytochrome c 6 from the cyanobacterium Anabaena sp. PCC 7119. Biochem Biophys Res Commun 243:302-306. doi:10.1006/bbrc.1997.7953

Molina-Heredia FP, Díaz-Quintana A, Hervás M, Navarro JA, De la Rosa MA (1999) Site-directed mutagenesis of cytochrome c 6 from Anabaena Species PCC 7119. J Biol Chem 274:33565-33570. doi:10.1074/jbc.274.47.33565

Molina-Heredia FP, Hervás M, Navarro JA, De la Rosa MA (2001) A single arginyl residue in plastocyanin and in cytochrome c 6 from the cyanobacterium Anabaena sp. PCC 7119 is required for efficient reduction of photosystem I. J Biol Chem 276:601-605. doi:10.1074/jbc.M007081200 
Molina-Heredia FP, Balme A, Hervás M, Navarro JA, De la Rosa MA (2002) A comparative structural and functional analysis of cytochrome c M, cytochrome c 6 , and plastocyanin from the cyanobacterium Synechocystis sp. PCC 6803. FEBS Lett 517:50-54. doi:10.1016/S0014-5793(02)02576-0

Molina-Heredia FP, Wastl J, Navarro JA, Bendall DS, Hervás M, Howe CJ, De la Rosa MA (2003) Photosynthesis: a new function for an old cytochrome? Nature 424:33-34. doi:10.1038/424033b

Muro-Pastor AM, Valladares A, Flores E, Herrero A (2002) Mutual dependence of the expression of the cell differentiation regulatory protein HetR and the global nitrogen regulator NtcA during heterocyst development. Mol Microbiol 44:1377-1385. doi:10.1046/j.1365-2958.2002.02970.x

Navarro JA, Durán RV, De la Rosa MA, Hervás M (2005) Respiratory cytochrome c oxidase can be efficiently reduced by the photosynthetic redox proteins cytochrome c 6 and plastocyanin in cyanobacteria. FEBS Lett 579:3565-3568. doi:10.1016/j.febslet.2005.05.034

Robertson EF, Dannelly HK, Malloy P, Reeves HC (1987) Rapid isoelectric focusing in a vertical polyacrylamide minigel system. Anal Biochem 167:290-294. doi:10.1016/0003-2697(87)90166-7

Schmetterer G (1994) Cyanobacterial respiration. In: Bryant DA (ed) The molecular biology of cyanobacteria. Kluwer Academic Publishers, Dordrecht, pp 409-435

Sommer F, Drepper F, Haehnel W, Hippler M (2004) The hydrophobic recognition site formed by residues PsaATrp651 and PsaB-Trp627 of photosystem I in Chlamydomonas reinhardtii confers distinct selectivity for binding of plastocyanin and cytochrome c 6. J Biol Chem 279:20009-20017. doi:10.1074/jbc.M313986200

Soriano GM, Ponomarev MV, Piskorowski RA, Cramer WA (1998) Identification of the basic residues of cytochrome $\mathrm{f}$ responsible for electrostatic docking interactions with plastocyanin in vitro: relevance to the electron-transfer reaction in vivo. Biochemistry 37:15120-15128. doi:10.1021/bi9807714

Ullmann GM, Hauswald M, Jensen A, Kostic NM, Knapp E-W (1997) Comparison of the physiologically equivalent proteins cytochrome c 6 and plastocyanin on the basis of their electrostatic potentials. Tryptophan 63 in cytochrome c 6 may be isofunctional with tyrosine 83 in plastocyanin. Biochemistry 36:16187-16196. doi:10.1021/bi971241v

Watkins JA, Cusanovich MA, Meyer TE, Tollin G (1994) A "parallel plate" electrostatic model for bimolecular rate constants applied to electron transfer proteins. Protein Sci 3:2104-2114. doi:10.1002/pro.5560031124

Weigel M, Varotto C, Pesaresi P, Finazzi G, Rappaport F, Salamini F, Leister D (2003) Plastocyanin is indispensable for photosynthetic electron flow in Arabidopsis thaliana. J Biol Chem 278:31286-31289. doi:10.1074/jbc.M302876200

Yu NY, Wagner JR, Laird MR, Melli G, Rey S, Lo R, Dao P, Sahinalp SC, Ester M, Foster L, Brinkman FSL (2010) PSORTb 3.0: Improved protein subcellular localization prediction with refined localization subcategories and predictive capabilities for all prokaryotes. Bioinformatics 26:1608-1615. doi:10.1093/bioinformatics/btq249

Zhang L, Pakrasi HB, Whitmarsh J (1994) Photoautotrophic growth of the cyanobacterium Synechocystis sp. PCC 6803 in the absence of cytochrome c 553 and plastocyanin. J Biol Chem 269:5036-5042 


\section{Figure captions}

Figure 1. UV/Visible absorption spectra of the purified Cyt c 6-like protein from Nostoc sp. PCC 7119 expressed in E. coli cells. The heme protein is shown both in its native oxidized state (dashed line) and after reduction by an excess of sodium dithionite (continuous line)

Figure 2. Alignment of the amino acid sequences of pre-cytochromes c 6 (c 6) and c 6-like protein (c 6-like) from Nostoc sp. PCC 7119 (a), and mature regions of Cyt c 6-like protein (c 6-like) from the cyanobacteria Nostoc sp. PCC 7119, N. spumigena CCY9414 and S. elongatus PCC 6301, Cyt c 6 (c 6) from the cyanobacteria Nostoc, S. elongatus PCC 6301 and Synechocystis sp. PCC 6803, and Cyt c 6A (c 6A) from the plants Arabidopsis thaliana var Columbia and Hordeum vulgare and from the green algae Chlamydomonas reinhardtii (b). Homologous regions are depicted in gray, whereas identical regions are boxed

Figure 3. Phylogenetic tree of His-Met Fe coordinated soluble Cytochromes from photosynthetic organisms. Trees were constructed by protein maximum likelihood with PROTML (MOLPHY) (Adachi and Hasegawa 1992). The following sequences were aligned: Cyt c 6-like protein (c 6-like) of Nostoc sp. PCC 7119 (EMBL AM902496), N. spumigena CCY9414 (GenBank gi 119509989), S. elongatus PCC 6301 (GenBank gi 56751577); Cyt c 6 (c 6) of Cladophora glomerata (GenBank gi 24636293), Chlorella vulgaris (GenBank gi 30578153), Chlamydomonas reinhardtii (GenBank gi 117924), Gloeobacter violaceus (GenBank gi 37521549), Monoraphidium braunii (GenBank gi 729268), Nostoc sp. PCC 7119 (EMBL AJ002361), S. elongatus PCC 6301 (GenBank gi 25014058), Synechocystis sp. PCC 6803 (EMBL P46445); mature Cyt c 6A (c A) of Aegilops speltoides (GenBank gi 11222607), Arabidopsis thaliana (GenBank gi 9783643), Medicago trunculata (GenBank gi 11610049), Oryza sativa (GenBank gi 8955747), Hordeum vulgare (GenBank gi 16311393), Solanum tuberosum (GenBank gi 13615108), Chlamydomonas reinhardtii (C_820065;); Cyt c M (c M) of Gloeobacter violaceus (GenBank gi 37522351), Nostoc sp. PCC 7120 (gi 17228860), Prochlorococcus marinus subsp. pastoris CCMP1378 (GenBank gi 33861613), Prochlorothrix hollandica (GenBank gi 4098525), S. sp. WH 8102 (GenBank gi 33865254), Synechocystis sp. PCC 6803 (GenBank gi 16330764); respiratory Cyt c (c) of Oryza sativa (GenBank gi 2394300), Chlamydomonas reinhardtii (GenBank gi 322374), Enteromorpha intestinalis (GenBank gi 65520), Solanum tuberosum (GenBank gi 65506); and Cyt c 2 (c 2) of Rhodopseudomonas palustris (TrEMBL Q8G180), Rhodospirillum centenum (TrEMBL P81153), Rhodovulum sulfidophilum (TrEMBL Q93163), Rhodobacter capsulatus (TrEMBL P00094), Roseobacter denitrificans (TrEMBL P07625), and Bradyrhizobium sp. ORS278 (TrEMBL Q8VUB4). Branch lengths reflect the estimated number of substitutions/site

Figure 4. Surface electrostatic potential distribution of Cyt c 6, Cyt c 6-like protein, and Pc from Nostoc sp. PCC 7119, and Cyt c 6-like protein from N. spumigena CCY9414 and S. elongatus PCC 6301. The heme group is depicted in yellow. All Cytochromes are represented in the same orientation with their respective "east" faces (site 2) in front and the north hydrophobic poles (site 1) at the top. Simulations of surface electrostatic potential distribution were performed assuming an ionic strength of $50 \mathrm{mM}$ at $\mathrm{pH}$ 7.0. Positively and negatively charged regions are depicted in blue and red colors, respectively

Figure 5. Dependence upon electron donor protein concentration of the observed pseudo-first-order rate constant (k obs) for PSI reduction by Cyt c 6 -like protein (filled circle), Cyt c 6 (unfilled circle) and Pc (filled square) from Nostoc sp. PCC 7119. The temperature was $25^{\circ} \mathrm{C}$ and the buffer used throughout this work was $20 \mathrm{mM}$ Tricine/KOH, pH 7.5, supplemented with $10 \mathrm{mM} \mathrm{MgCl2}$. The oscilloscope traces obtained with the Cyt c 6 molecule fit to biphasic kinetics, whereas those corresponding to Cyt c 6 -like protein and Pc fit to single exponential curves 
Figure 6. Effect of ionic strength on $\mathrm{k}$ bim for PSI reduction by Cyt c 6 -like protein (filled circle), Cyt c 6 (unfilled circle) and Pc (filled square) from Nostoc sp. PCC 7119. Experimental conditions were as described in the legend to Fig. 4, except for magnesium chloride that was omitted from the reaction mixture. The ionic strength was adjusted to the desired value by adding small amounts of a concentrated $\mathrm{NaCl}$ solution. Effect of ionic strength on $\mathrm{k}$ bim for PSI reduction by Cyt c 6-like protein (filled triangle). The ionic strength was adjusted to the desired value by adding small amounts of a concentrated $\mathrm{MgCl} 2$ solution. Continuous lines represent theoretical fits according to the formalism developed by Watkins et al. (1994) 
Table 1

Physicochemical and functional properties of Nostoc sp. PCC 7119 Cyt c ${ }_{6}$, Cyt c ${ }_{6}$-like protein, and Pc

\begin{tabular}{|c|c|c|c|c|c|c|}
\hline Protein & $\begin{array}{l}\text { Mass } \\
(\mathbf{k D a})\end{array}$ & $\begin{array}{l}\text { Isoelectric } \\
\text { (pI) }\end{array}$ & \begin{tabular}{l|l} 
point & $E_{m}(m V)$ at $p H$ \\
7.0
\end{tabular} & $\begin{array}{l}\mathbf{k} \\
\left(M^{-1} s^{-1}\right)^{a}\end{array}$ & $\operatorname{bim} \frac{k}{\left(M^{-1} s^{-1}\right)^{b}}$ & $\inf \mathbf{k}_{\text {et }}\left(\mathbf{s}^{-1}\right)^{\mathbf{c}}$ \\
\hline Cyt c 6 & 9.7 & 9.0 & +335 & $12.1 \times 10^{7}$ & $11.4 \times 10^{6}$ & $1.7 \times 10^{5}$ \\
\hline $\begin{array}{lll}\text { Cyt } & \text { c } & 6^{-} \\
\text {like } & & \end{array}$ & 10.2 & 8.0 & +199 & $3.2 \times 10^{7}$ & $4.0 \times 10^{6}$ & \\
\hline Pc & 11.1 & 8.8 & +357 & $7.6 \times 10^{7}$ & $5.9 \times 10^{6}$ & \\
\hline
\end{tabular}

${ }^{\mathrm{a}} \mathrm{k}$ bim is the second-order rate constant for PSI reduction

${ }^{\mathrm{b}} \mathrm{k}_{\text {inf }}$ is the $\mathrm{k}_{\text {bim }}$ extrapolated to infinite ionic strength

${ }^{c} \mathrm{k}_{\text {et }}$ is the first-order electron transfer rate constant for PSI reduction 
Figure 1




Figure 2




Figure 3






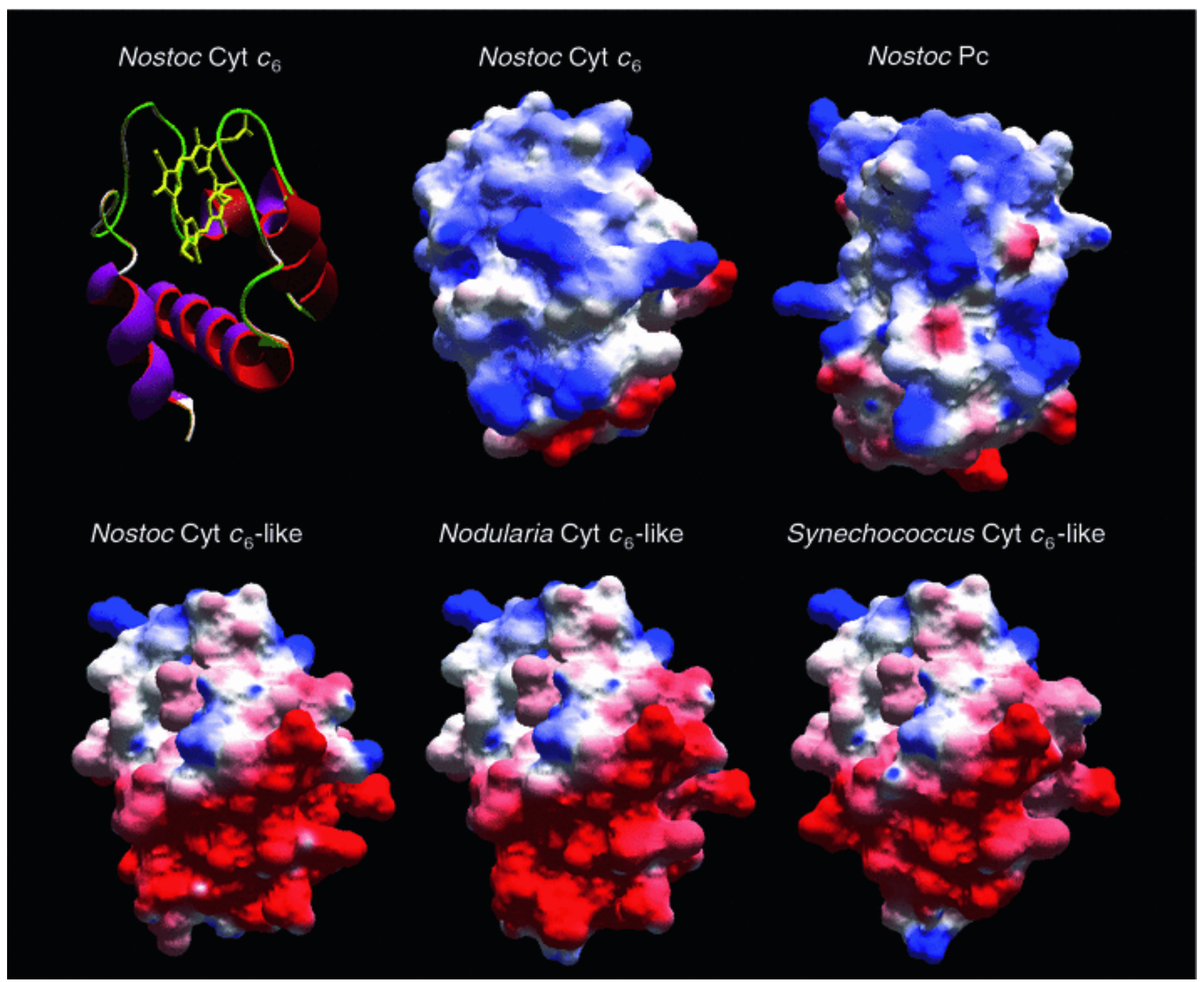


Figure 5

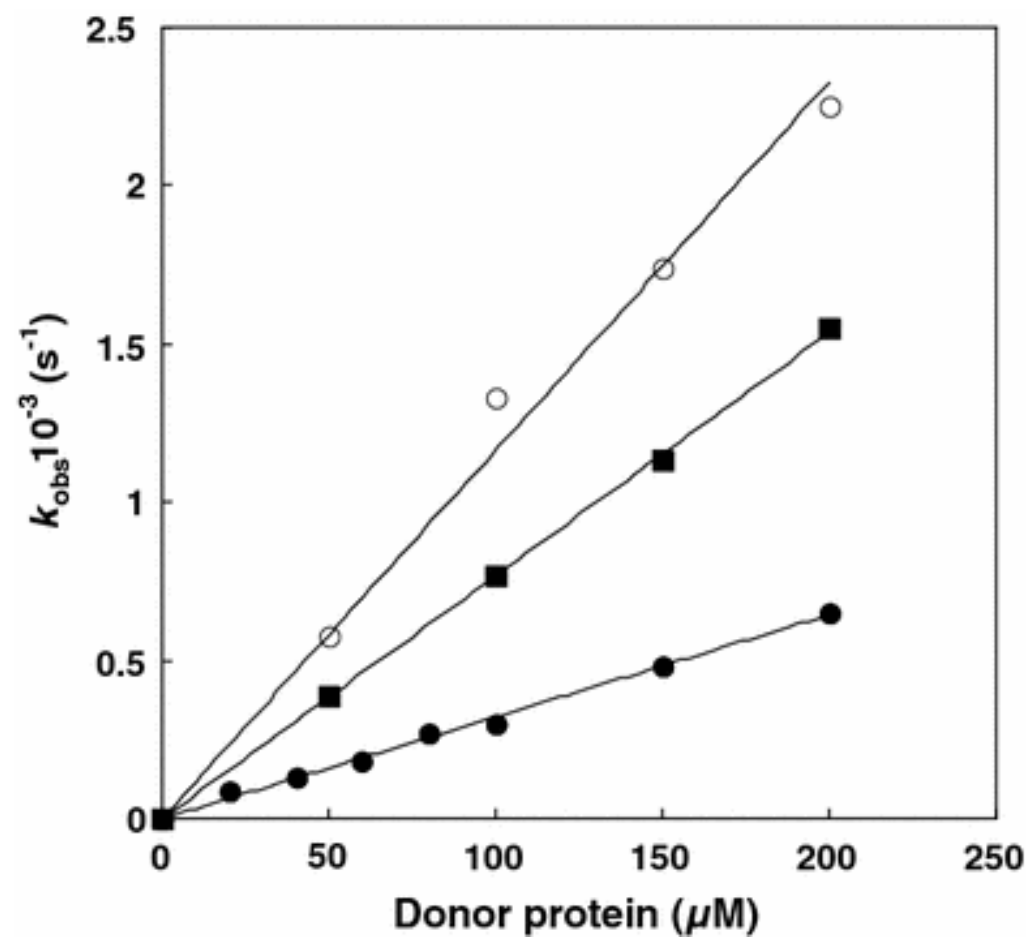


Figure 6



